\title{
The Further of Unpowered Exoskeleton Assist Robot in Rehabilitation
}

\author{
Lei Jiang ${ }^{1 *}$, Zhenjing $\mathrm{Li}^{1,2}$, Yang Liu ${ }^{1}$, Yujuan Song ${ }^{1,2}$, and Christoph Korallus ${ }^{1}$ \\ ${ }^{1}$ Department of Rehabilitation, Hannover Medical School, Germany \\ ${ }^{2}$ Department of Rehabilitation, Longhua District Central Hospital, China
}

*Corresponding author: Lei Jiang, Department of Rehabilitation, Hannover Medical School, Hannover Germany.

To Cite This Article: Lei Jiang, The Further of Unpowered Exoskeleton Assist Robot in Rehabilitation. 2020 - 8(1). AJBSR.MS.ID.001229.

DOI: 10.34297/AJBSR.2020.08.001229.

Received: 阱 February 29, 2019; Published: March 10, 2020

\section{Exoskeleton Assist Robot-With or Without Power?}

Exoskeleton assist robot is widely used in rehabilitation medicine, it has an irreplaceable function in patient disability functional recovery, and in studies too. Most exoskeleton robot are powered by electric, hydraulic or pneumatic, as a power source, meanwhile, the presence of high quality, high cost, inconvenient to use and other issues are also not easy to solve. At the same time, energy and battery life issues are also important factors affecting the development of exoskeleton-assisted robots [1]. However, unpowered exoskeleton assist robot needs no additional power, it does not need energy equipment or other unnatural external forces to drive, except the user's energy provided to storage and release [2]. Compared with the traditional powered exoskeleton, the unpowered exoskeleton robot has the advantages of smaller mass and no energy consumption and solves the long-term shortcomings of the powered exoskeleton assisted robot.

Despite this, exoskeleton assist robot without power, it is exoskeleton, also it is hard to be considered a robot, although as early as 1830, Robert Seymour had put forward the idea of wearing the steam engine power. Robots are not products that Steampunk can outline; it must at least with basic artificial intelligence as a hub for computing assistance. There is no doubt that light weighting of powered exoskeleton robots is one of the development trends in mainstream academic circles; in the meantime, empowering unpowered exoskeleton robots with artificial intelligence, within the range of minimizing power input, is also one of the development directions.

\section{Unpowered Exoskeleton Boosting Principle and Developed Produces}

The principle of unpowered exoskeletons is based on human anatomy and biology and aims to reproduce the mechanism of the human skeletal muscle system [3]. The human skeletal muscle system is the physiological material basis for the realization of various movements of the human body and is the basis for the dynamic interaction between the human body and the outside world. Skeletal muscles are the power source for human movement. Tendons connect muscles to bones. Muscles contract by pulling tendons to produce joint movements [4].

Exoskeleton unpowered assist robot is mainly composed of mechanical support, a powerless elastic element, and connecting piece, corresponding to the bones, muscles, and tendons in the human skeletal muscle, it is a perfect combination of bionics, dynamics, and mechanical engineering. The spring simulates the cooperation of human muscles and tendons to store, release, and conduct deformation potential energy [5], recycle the energy during human movement, thereby reducing the wearer's own energy consumption; on the other hand, researchers design a scientific exoskeleton structure based on the interaction force between the exoskeleton and the human body, the rational arrangement of the mechanical structure can reasonably transmit the force [6], the "unpowered" requirement in the unpowered exoskeleton is thus achieved.

At present, fluid springs, scroll springs, shape memory alloy, elastic soft material, etc. are often used in unpowered exoskeleton assist robot [7]. Many studies have pointed out that unpowered exoskeleton assist produces can provide 13\%-60\% strength improvement in supporting the erector spinae muscles and knee joints. Ekso Bionics company and Levitate AIRFRAME in California studied a lot in upper limb wearable unpowered exoskeleton, meanwhile lower extremity wearable unpowered exoskeleton, independent joint unpowered exoskeleton, torso support unpowered exoskeleton are well developed as well. 


\section{The Intelligent Trend of Unpowered Exoskeleton Assist Robot}

As it showed at the beginning, the low intelligent is the congenital shortcoming in unpowered exoskeleton device, as its pursuit of lightweight. However, the most of the wearables' weight lies in the processor and battery, the same situation for powered exoskeleton robots. Therefore, unpowered exoskeleton assist robot can drop the bulky batteries, processors and boards, leaving only lightweight sensors and micro batteries for sensor operation, and sending data to a remote server. The widespread application of Internet of Things (IoT) chips has made this design a reality. The unpowered exoskeleton assist produces plus IoT sensors, with high-speed network transmission technology, the user's data can be transported to the with extremely low network latency. The process structure is as Figure 1.

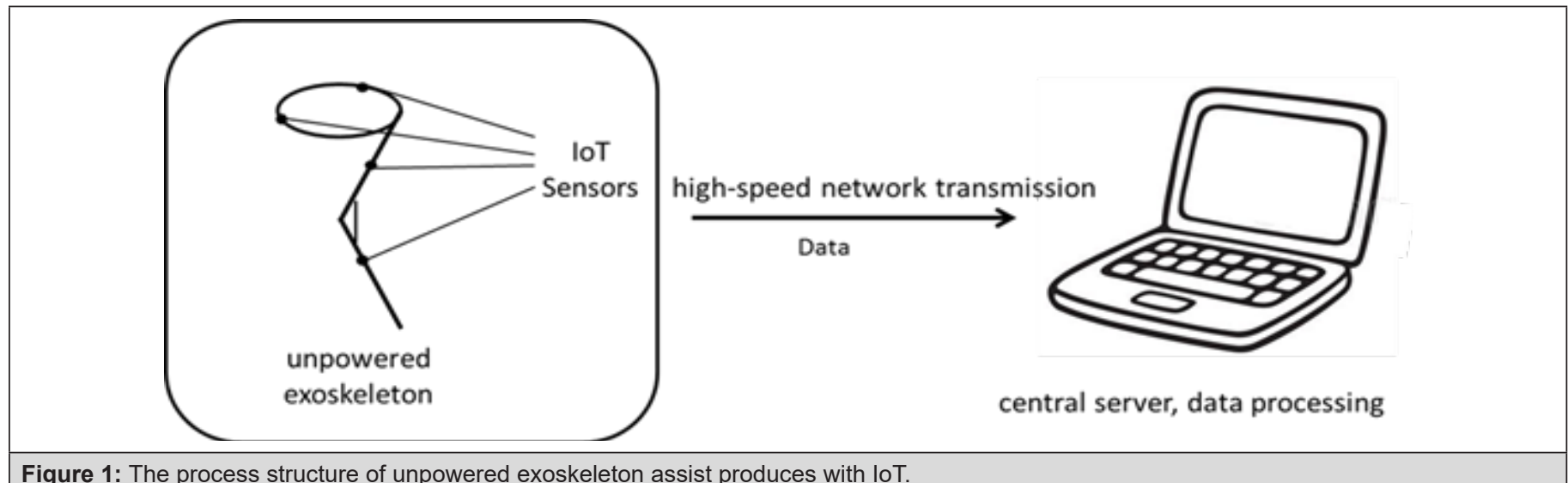

Figure 1: The process structure of unpowered exoskeleton assist produces with loT.

The IoT sensors can record data of mechanical structure stability, load capacity, wear coordination, requirements for precise control, the involved sensors include high precision $3 \mathrm{~d}$ gravity acceleration sensors, photoelectric sensors, sound sensors, gyro sensors, etc. These data are processed by the central processor and fed back to the rehabilitation physician, in order to grasp the user's, and then make further adjustments to equipment to make it more suitable for use, be truly vary, personalized treatment. The above design and ideas have been implemented in our scientific research.

\section{References}

1. Gopura R, Kiguchi k (2009) Mechanical designs of active upper-limb exoskeleton robots state-of-the-art and design difficulties. Interna confea Rehabilitation Robotics. New York pp.178-187.

2. Kolakowsky H, Stephanie (2013) A Safety and feasibility of using the eksotm bionic exoskeleton to aid ambulation after spinal cord injury. journal of spine $4: 8-9$
3. Si W, Srivastava G, Zhang Y, Jiang L (2019) Green internet of things application of a medical massage robot with system interruption. In IEEE Access 7: 127066-127077.

4. Bortoletto R, Sartori M, He Fuben, Enrico P (2012) Simulating an elastic bipedal robot based on musculoskeletal modeling. Living machines 7375: 26-37

5. Yheng chao (2016) Study on low energy consumption driven joint of exo-skeleton based on energy flow characteristics of human body. Harbin institute of technology p.10-15

6. Ulrey BL, Fathallah FA (2013) Effect of a personal weight transfer device on muscle activities and joint flexions in the stooped posture. J Electromyogr Kinesiol 23(1): 195-205.

7. Lai Qizi, Chen Suyang, Zhang Yuling, (2015) Design of a trainer utilizing the torsion of spiral spring for ankle Rehabilitation. China Health Care Nutrition 25(13): 38-39. 